\title{
Development Oral Available Melanotropins Based on the Cyclic Peptide Framework of Sunflower Trypsin Inhibitor-1
}

\author{
Minying Cai ${ }^{1}$, David Craik ${ }^{2}$, Horst Kessler ${ }^{3}$, and Victor J. Hruby ${ }^{1}$ \\ ${ }^{1}$ Department of Chemistry and Biochemistry, The University of Arizona, Tucson, AZ, 85721, USA; ${ }^{2}$ Queensland \\ Tropical Health Alliance, School of Pharmacy and Molecular Sciences and Centre for Biodiscovery and \\ Molecular Development of Therapeutics, James Cook University, Cairns, QLD 4870, Australia $;{ }^{3}$ Center of \\ Integrated Protein Science Institute for Advanced Study at the Technische Universitat Munchen,
} Lichtenbergster, 4, 85747, Garching, Germany

\section{Introduction}

The melanocortin system involves numerous physiological functions and is associated with many diseases such as skin cancer, obesity and diabetes, sexual dysfunction, neuropathic pain, inflammatory diseases etc. Our primary interests have been focusing on the human melanocortin 1 and 4 receptors (hMC1R, hMC4R) due to their direct involvement in the regulation of feeding behavior, energy homeostasis, as well as inflammatory diseases. Nevertheless, the full scope of physiological functions of these receptors is still poorly understood. There has been a resurgence of interest in peptide pharmaceuticals recently as they have an advantage of potency, selectivity and less toxicity compared with small-molecule therapeutics. The main drawback of peptides is lack of stability in biological media. Using cycloids and N-Methylation have been useful options to improve in vivo stability of the peptides [1]. Several new modalities in constraining peptides have been developed over recent years and this work highlights some of the new developments in our lab [2,3]. The newer grafted and methylation strategies have rendered, in some cases, oral activity, cell permeability, improved potency at the target receptor, selectivity against receptor subtypes and improved stability to enzymes. Further understanding rules governing cell permeability, oral absorption and enhancing stability of peptides can help peptides to enter the clinic for many unmet medical needs.

\section{Results and Discussion}

During the last decade, great efforts have been made to develop selective melanotropins. Nevertheless, the potential of potent and selective peptides as drug candidates is challenged by their poor pharmacokinetic properties. Many peptides have a short half-life in vivo and a lack of oral availability. Inspired by the excellent pharmacokinetic profile of cyclosporine, a natural, multiply $\mathrm{N}$-methylated cyclic peptide, we visualized using cycloid as a stable template and grafted with multiple $\mathrm{N}$-methylated pharmacophore of NDP- $\alpha-\mathrm{MSH}$, His-D-Phe-Arg-Trp, as a promising way to rationally improve key pharmacokinetic characteristics of melanotropins. The incorporation of this strategy to multiple Nmethylated analogs of melanotan II (MTII) has successfully reached this goal by increasing receptor selectivity and peptide stability [4]. Here, we extended our efforts toward a grafted cycloid sunflower trypsin inhibitor-1, SFTI-1, to develop novel oral available melanocortin receptor agonists and antagonists. The melanocortin 1 receptor $(\mathrm{hMC} 1 \mathrm{R})$ selective ligand has great potential to prevent melanoma. We aimed to develop selective and orally available melanotropins by applying the pharmacophore of NDP- $\alpha$-MSH, His-D-Phe-Arg-Trp, sequence into the SFTI-1 framework. Combined with multiple N-Methylation strategy, thirty peptides were synthesized using solid phase peptide chemistry and $\mathrm{H} \alpha$ NMR chemical shifts were used to confirm that the structures of the peptides were similar to native SFTI-1. The analogs were tested in a series of binding and functional assays at melanocortin receptors 1 and 3, 4, 5 and several peptides showed potent agonist or antagonist activity. We discovered that several selective hMC1R agonists and hMC4R agonists [5]. As an example cyclo[C-T-A-S-I-P-P-I-C-H-f-R-W-R] is a nano-molar selective peptide for hMC1R (Table 1). In this peptide Arginine (R) and Tryptophan (W) are N-Methylated (Grey highlighted amino acids). The binding efficacy is showing $100 \%$ and the binding affinity is showing $48 \mathrm{nM}$, while the other types of melanocortin receptors showing no binding affinity. 


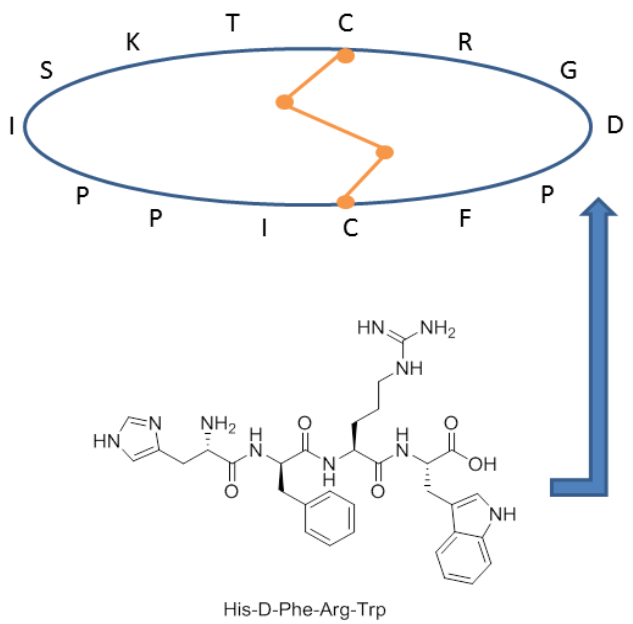

Fig. 1. Primary sequence of the SFTI-1 scaffold (Top), the disulfide bond is shown in yellow; Structure of the grafting pharmacophore of melanotropin (His-D-Phe-Arg-Trp) (Bottom).

The improvement of oral bioavailability by multiple $\mathrm{N}$-methylation and using cycloid template are significant advances toward the development of peptide-based therapeutics, which has been hampered over the years due to poor pharmacokinetic properties. Cycloid grafted peptides combined with multiple N-methylation of NDP- $\alpha-\mathrm{MSH}$ pharmacophore result in enhancement in the activity and selectivity of receptor subtypes using either library or designed approaches and helps in understanding the finer details of the bioactive conformation. Thus, with these diverse properties, we foresee a bright future for peptide chemistry by multiple $\mathrm{N}$-methylation toward their development as therapeutic prototypes.

Table 1. Binding assay of N-methylated SHU9119 analogues at hMCRs.

\begin{tabular}{cccccccccc}
\hline & \multicolumn{2}{c}{$h M C 1 R$} & \multicolumn{2}{c}{$h M C 3 R$} & \multicolumn{2}{c}{$h M C 4 R$} & \multicolumn{2}{c}{$h M C 5 R$} \\
\cline { 2 - 9 } & $\begin{array}{c}\mathrm{IC}_{50} \\
\mathrm{nM}\end{array}$ & $\mathrm{PBE}^{\mathrm{a}}$ & $\begin{array}{c}\mathrm{IC}_{50} \\
\mathrm{nM}\end{array}$ & $\mathrm{PBE}^{\mathrm{a}}$ & $\begin{array}{c}\mathrm{IC}_{50} \\
\mathrm{nM}\end{array}$ & $\mathrm{PBE}^{\mathrm{a}}$ & $\begin{array}{c}\mathrm{IC}_{50} \\
\mathrm{nM}\end{array}$ & $\mathrm{PBE}^{\mathrm{a}}$ \\
\hline MTII & $1 \pm 0.1$ & 100 & $2 \pm 0.2$ & 100 & $2.4 \pm 0.2$ & 100 & $6.9 \pm 1.2$ & 100 \\
cyclo[C-T-A-S-I-P-P-I-C-H-f-R-WW-R] & 48 & 100 & 4640 & 100 & $>10,000$ & 100 & $>10,000$ & $\mathrm{NB}$ \\
\hline
\end{tabular}

$I C_{50}=$ concentration of peptide at 50\% specific binding $(N=4) . N B=0 \%$ of ${ }^{125} I-N D P-\alpha-M S H$ displacement observed at $10 \mu \mathrm{M}$. ${ }^{a}$ Percent Binding Efficiency = maximal \% of ${ }^{125} \mathrm{I}-\mathrm{NDP}-\alpha-\mathrm{MSH}$ displacement observed at $10 \mu M$. Grey highlighted amino acids are N-methylated.

\section{Acknowledgments}

This work is supported in part by a grant from the U.S. Public Health Service, National Institutes of Health, DK017420, GM 108040 and DA06284.

\section{References}

1. Kessler, H. Angew. Chem. Int. Ed. 21, 512-523 (1992), http://dx.doi.org/10.1002/anie.198205121

2. Hruby, V.J. Nature Reviews Drug Discovery 1, 847-858 (2002), http://dx.doi.org/10.1038/nrd939

3. Wang, C. K., Gruber, C. W., Cemazar, M., Siatskas, C., Tagore, P., Payne, N., Sun, G., Wang, S., Bernard, C. C., Craik, D. J. (2014). 9: 156-163. http://dx.doi.org/10.1021/cb400548s

4. Doedens, L., Opperer, F., Cai, M., Beck, J.G., Dedek, M., Palmer, E., Hruby, V.J., Kessler H. J. Amer. Chem. Soc. 132, 8115-8128 (2010), http://dx.doi.org/10.1021/ja101428m

5. Hruby, V.J., Lu, D., Sharma, S.D., Castrucci, A.L., Kesterson, R.A., Al-Obeidi, F.A., Hadley, M.E., Cone, R.D. J. Med. Chem. 38, 3454-3461 (1995), http://dx.doi.org/10.1021/jm00018a005 